\title{
Transient Absorption Spectroscopy for Polymer Solar Cells
}

$\operatorname{AUTHOR}(\mathrm{S})$ :

Ohkita, Hideo; Tamai, Yasunari; Benten, Hiroaki; Ito, Shinzaburo

\section{CITATION:}

Ohkita, Hideo ... [et al]. Transient Absorption Spectroscopy for Polymer Solar Cells. IEEE Journal of Selected Topics in Quantum Electronics 2016, 22(1): 100-111

\section{ISSUE DATE:}

2016-01

URL:

http://hdl.handle.net/2433/209995

\section{RIGHT:}

(C) 2015 IEEE. Personal use of this material is permitted. Permission from IEEE must be obtained for all other uses, in any current or future media, including reprinting/republishing this material for advertising or promotional purposes,

creating new collective works, for resale or redistribution to servers or lists, or reuse of any copyrighted component of this work in other works.; This is not the published version. Please cite only the published version.; この論文は出版社版

でありません。引用の際には出版社版をご確認ご利用ください。 


\title{
Transient Absorption Spectroscopy for Polymer Solar Cells
}

\author{
Hideo Ohkita, Yasunari Tamai, Hiroaki Benten, and Shinzaburo Ito
}

\begin{abstract}
Time-resolved spectroscopy is a powerful tool for studying fundamental photophysics in optoelectronic materials on a molecular temporal scale. In this review, we describe transient spectroscopic studies on fundamental photovoltaic conversion processes in polymer solar cells, which consist of a series of conversion processes such as photon absorption, exciton diffusion into a donor/acceptor interface, charge transfer at the interface, charge dissociation into free charge carriers, and charge collection to each electrode. These conversion processes are ultrafast phenomena and are ranging over the wide temporal scale from femtoseconds to microseconds, which can be directly observed by transient spectroscopy.
\end{abstract}

Index Terms-Transient spectroscopy, polymer solar cells, exciton, singlet fission, geminate recombination, non-geminate recombination

\section{INTRODUCTION}

$\mathrm{T}$ RANSIENT spectroscopy is a powerful tool for tracing ultrafast phenomena directly in real time. Historically, Norrish and Porter developed the flash photolysis technique around 1950 before the invention of laser (light amplification by stimulated emission of radiation) [1,2]. Their pioneering study opened a new world of research in a microsecond time domain, which enables us to detect short-lived transient species directly. As a result, they were awarded the Nobel Prize in Chemistry 1967 [3,4]. Subsequently, this technique has been further improved in temporal resolution with the emergence of short-pulsed lasers. Currently, one can discuss ultrafast phenomena with a resolution of femtoseconds. In 1999, Zewail was awarded the Nobel Prize in Chemistry for his studies of the transition states of chemical reactions using femtosecond spectroscopy [5]. From the establishment of the flash photolysis technique, the temporal resolution has been reduced by about eight orders of magnitude with shortening laser pulse duration. Nowadays, as a result, transient spectroscopy has been widely employed in various research fields in order to directly detect short-lived intermediate species over the wide temporal range from seconds up to femtoseconds. In this review, we focus on each photovoltaic conversion process in polymer solar cells, which includes photon absorption (exciton

This work is supported in part by the JST PRESTO program (Photoenergy and Conversion System and Materials for the Next Generation Solar Cells) and JSPS KAKENHI (Grant-in-Aid for Scientific Research (A), No. 26248033). H. Ohkita is with the Department of Polymer Chemistry, Graduate School of Engineering, Kyoto University, Kyoto 615-8501, Japan, and also with the Japan Science and Technology Agency, PRESTO, Saitama 332-0012, Japan generation), exciton diffusion into a donor/acceptor interface, charge transfer at the interface, charge dissociation into free charge carriers, and charge collection to each electrode as shown in Fig. 1 [6-17]. The series of conversion processes are typically ranging from femtoseconds to microseconds. Thus, transient spectroscopy is the most suitable and useful method for studying such ultrafast conversion processes in real time.

\section{Transient Absorption MEASUREMEnTS}

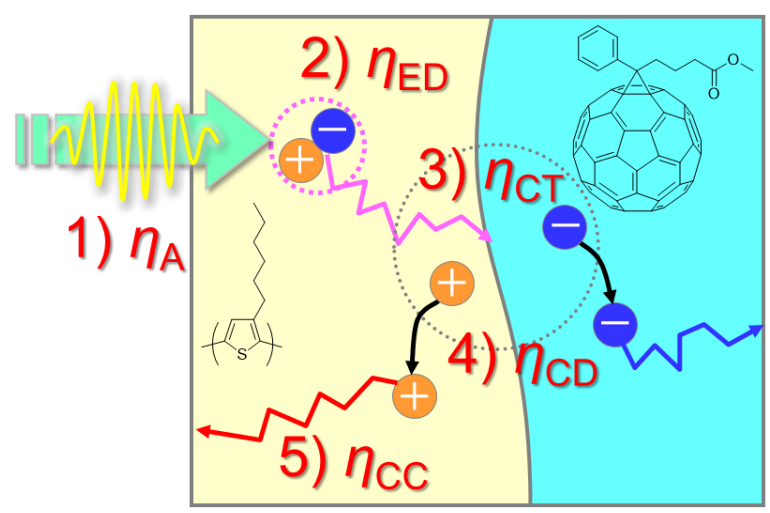

Fig. 1. Photovoltaic conversion processes in polymer solar cells: 1) photon absorption (exciton generation), 2) exciton diffusion into a donor/acceptor interface, 3) charge transfer at the interface, 4) charge dissociation into free charge carriers, and 5) charge collection to each electrode.

Transient absorption spectroscopy is one of the most useful and powerful methods for observing transient species such as excitons and charge carriers generated by photoexcitation. In the transient absorption measurement, the probe light intensity transmitted through a sample is detected before $I_{0}$ and after $I$ the photoexcitation. From the intensity ratio, the absorbance change is given by $\Delta \mathrm{OD}=\log \left(I_{0} / I\right)$. Consequently, photoproducts provide positive signals in the $\triangle \mathrm{OD}$ but emission or photobleaching due to the decrease in the ground state results in negative signals in the $\Delta \mathrm{OD}$. According to the LambertBeer's law, on the other hand, the absorbance change is given by the product of a molar absorption coefficient $\varepsilon$, a molar concentration $c$, and an optical path length $l(\Delta \mathrm{OD}=\varepsilon c l)$. It is

(e-mail: ohkita@photo.polym.kyoto-u.ac.jp)

Y. Tamai, H. Benten, and S. Ito are with the Department of Polymer Chemistry, Graduate School of Engineering, Kyoto University, Kyoto 615 8501, Japan (e-mail: tamai@ photo.polym.kyoto-u.ac.jp; benten@photo.polym.kyoto-u.ac.jp; sito@photo.polym.kyoto-u.ac.jp). 
therefore technically difficult to detect a small absorbance change of thin films such as polymer solar cells, in which the photoactive layer is typically as thin as $100 \mathrm{~nm}\left(=10^{-5} \mathrm{~cm}\right)$. For such thin films $\left(l=10^{-5} \mathrm{~cm}\right)$, the absorbance would be as small as $10^{-5}$ for a typical condition of $\varepsilon=10^{4} \mathrm{M}^{-1} \mathrm{~cm}^{-1}$ and $c=0.1$ $\mathrm{mM}$. To detect such an extremely small absorbance change of $10^{-5}$, we need to measure only $1 / 50000$ of the change in the optical probe signal separately from various noises.

For ultrafast phenomena on a time scale of $<1 \mathrm{~ns}$, the pump and probe technique has been widely employed. In this technique, ultrashort laser pulses are divided into two pulses: one serves as a pump light for the sample excitation and the other serves as a probe light for the transmittance measurement. By controlling the arrival time of the pump and probe laser pulses at the sample, transient absorption can be measured at various delay times. The delay time can be tuned with an optical delay line: the probe light is delayed relative to the pump light because of the additional path length in the optical delay line. For example, when the total optical delay length is set at $30 \mathrm{~cm}$ the probe light is delayed by $1 \mathrm{~ns}$ relative to the pump light, and therefore the $\Delta \mathrm{OD}$ at $1 \mathrm{~ns}$ after the laser excitation can be measured. Fig. 2 shows a block diagram of a typical pump and probe spectroscopy system we employ [18-28]. This system consists of an ultrafast pulsed laser, a wavelength converter, and a pump and probe spectrometer. As shown in the figure, the amplified Ti:sapphire laser provides $800-\mathrm{nm}$ fundamental pulses at a repetition rate of $1 \mathrm{kHz}$ with an energy of $0.8 \mathrm{~mJ}$ and a pulse width of $100 \mathrm{fs}$ (FWHM), which are split into two optical beams with a beam splitter to generate pump and probe pulses. One fundamental beam is converted into pump pulses at $400 \mathrm{~nm}$ with a second harmonic generator or pump pulses at other wavelengths with an ultrafast optical parametric amplifier. The other fundamental beam is converted into white light continuum pulses employed as probe pulses at wavelengths ranging from 400 to $1700 \mathrm{~nm}$. The pump pulses are modulated mechanically with a repetition rate of $500 \mathrm{~Hz}$. The temporal evolution of the probe intensity is recorded with a CMOS linear sensor for the visible measurement and with an InGaAs linear diode array sensor for the near-IR measurement.

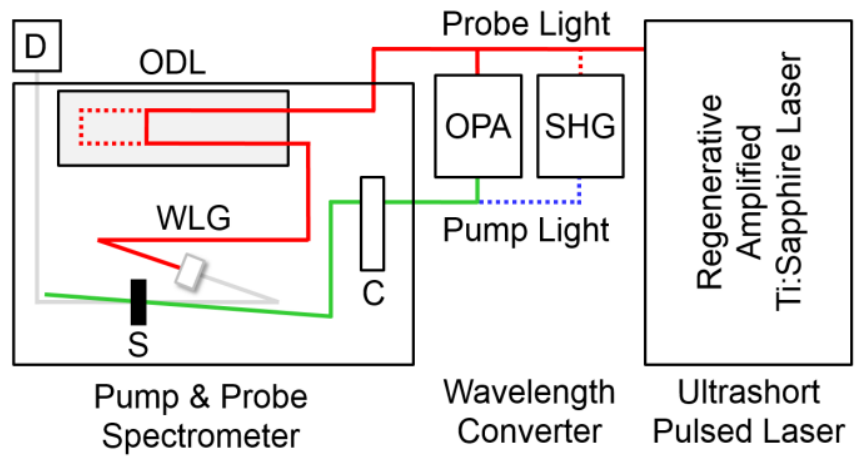

Fig. 2. Block diagram of a pump and probe femtosecond transient absorption measurement system, which consists of ultrashort pulsed laser, wavelength converter, and pump and probe spectrometer: SHG second harmonic generator, OPA optical parametric amplifier, C chopper, ODL optical delay line, WLG white light generator, S sample, and D detector. The detector is replaceable: a CMOS linear sensor for the visible wavelength range and an InGaAs linear diode array sensor for near-IR wavelength range.
Transient absorption spectra and decays are collected over the time range from $-5 \mathrm{ps}$ to $3 \mathrm{~ns}$. Typically, 2500 laser shots are averaged on each delay time to obtain a detectable absorbance change as small as $10^{-4}-10^{-3}$ depending on the monitoring wavelength. The polarization direction of the linearly polarized probe pulse is set at a magic angle of $54.7^{\circ}$ with respect to that of the pump pulse to cancel out orientation effects on the dynamics. In order to measure transient absorption on later time range, a longer optical delay line would be required. Alternately, an additional laser is employed to provide delayed probe pulses with an electric delay generator synchronized with the pump laser pulse. In such a case, it is necessary to correct signal jitters due to the electric circuit.

At a much later time stage, a highly sensitive transient absorption spectroscopy system is required because most transient species already decay significantly. Fig. 3 shows a block diagram of the highly sensitive microsecond transient absorption spectroscopy system [18,26,29-31]. In this system, a probe light is provided from a tungsten lamp with a power source stabilized to reduce fluctuation of the probe light intensity. To reduce scattering light, stray light, and emission from the sample, two monochromators and appropriate optical cut-off filters are placed both before and after the sample. An excitation light is supplied from a dye laser pumped by a nitrogen laser, which can excite an absorption peak of thin-film samples to give a high yield of photoexcitations. The probe light passing through the sample is detected with a PIN photodiode such as Si or InGaAs depending on the measuring wavelength. The signal from the photodiode is pre-amplified and sent to the main amplification system with electronic bandpass filters to improve the signal to noise ratio. The amplified signal is collected with a digital oscilloscope, which is synchronized with a trigger signal of the laser pulse from a photodiode. With this system, the detectable absorbance change is as small as $10^{-5}$ to $10^{-6}$ depending on the measuring time domain after appropriate accumulation.

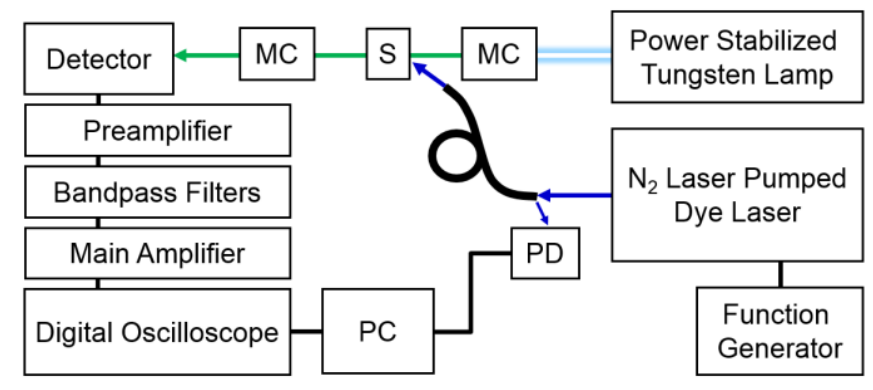

Fig. 3. Block diagram of highly sensitive microsecond transient absorption measurement system: MC monochromator, S sample, PC computer, PD PIN photodiode to detect a part of the pump laser light as a trigger signal, which is sent to the digital oscilloscope. The detector for the probe light is replaceable: Si PIN photodiode for the visible wavelength range and an InGaAs PIN photodiode for near-IR wavelength range. 


\section{EXCITON DYNAMICS}

\section{A. Exciton Diffusion}

Excitons are coulombically bound electron-hole pairs and hence cannot contribute to the photocurrent in themselves. To dissociate into free carriers, excitons should diffuse to a donor/acceptor interface where the energy offset in the lowest unoccupied molecular orbital (LUMO) or the highest occupied molecular orbital (HOMO) levels of the donor and acceptor materials is enough to break the Coulomb attraction. As such, the exciton diffusion dynamics and length have been intensively studied to design efficient exciton harvesting. For example, the exciton diffusion length has been reported to be 5-7 $\mathrm{nm}$ for poly ( $p$-phenylene vinylene) (PPV) films [32-37]. On the other hand, it has been reported to be 4-27 nm for regioregular poly(3-hexylthiophene) (RR-P3HT) films [38-44]. In other words, there is a good agreement for PPV amorphous films but considerable discrepancy for RR-P3HT crystalline films. This is probably because crystalline polymers generally consist of not only crystalline but also amorphous domains. Consequently, the exciton dynamics previously studied would be observed for both crystalline and amorphous domains with different ratios depending on the measuring conditions, resulting in different diffusion lengths.

The exciton diffusion length is typically evaluated by the following two methods. One is photoluminescence (PL) quenching in bilayer films based on exciton-generating donor layer and exciton-quenching acceptor layer. For crystalline donor polymers, the exciton diffusion evaluated by this method would be average one in crystalline and amorphous domains because the donor layer should have both domains. The other is singlet-singlet exciton annihilation measured by timeresolved spectroscopy such as transient absorption spectroscopy. Without selective excitation, excitons would be generated in both amorphous and crystalline domains and hence averaged dynamics would be observed. We therefore emphasize that selective excitation is of particular importance in order to study the exciton diffusion dynamics and length in crystalline domains.

We have studied the exciton diffusion dynamics and length in RR-P3HT crystalline domains with different crystallinity by analyzing singlet-singlet exciton annihilation dynamics upon selective excitation at $620 \mathrm{~nm}$ where the absorption is safely ascribed not to amorphous but to crystalline phase alone. Fig. 4 shows the transient absorption spectra of P3HT films with different crystallinity. Upon the selective excitation of RRP3HT at $620 \mathrm{~nm}$, as shown in Figs. 4a and 4b, singlet exciton band is observed at around $1300 \mathrm{~nm}$ immediately after the laser excitation, and then decays monotonically but is not shifted at all with time. In contrast, as shown in Fig. 4c, singlet exciton band is observed for regiorandom P3HT (RRa-P3HT) at 1050 $\mathrm{nm}$ immediately after the laser excitation, and then decays with time, being redshifted to longer wavelengths. These findings show that RR-P3HT crystalline domains are energetically homogeneous while RRa-P3HT amorphous films are energetically so inhomogeneous that excitons are likely to migrate to more stable sites with time. Upon the excitation of

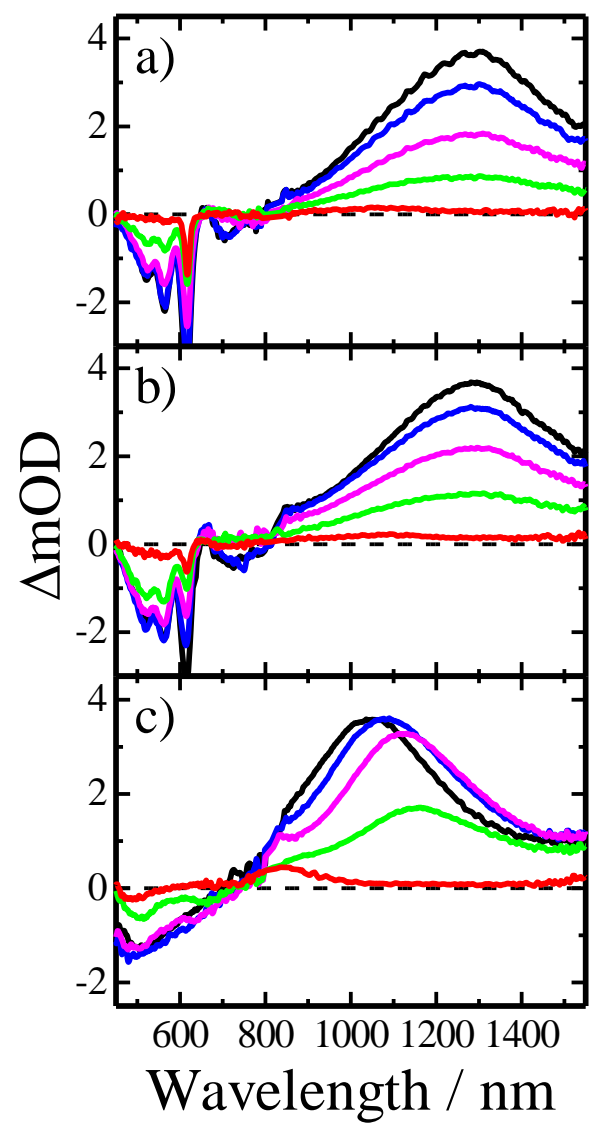

Fig. 4. Transient absorption spectra of P3HT films with different crystallinity measured at $0,1,10,100,1000 \mathrm{ps}$ after the laser excitation from top to bottom in each panel: a) RR-P3HT_H and b) RR-P3HT_L excited at $620 \mathrm{~nm}$ and c) RRa-P3HT at $400 \mathrm{~nm}$ [25].

RR-P3HT at $400 \mathrm{~nm}$, the singlet exciton band is redshifted with time as is the case with RRa-P3HT, suggesting that excitons are generated not only in crystalline but also in amorphous domains. In summary, the selective excitation is essential for correct understanding the exciton dynamics in crystalline domains.

The exciton diffusion dynamics can be discussed by analyzing the singlet-singlet exciton annihilation, which is dependent upon the excitation intensity. At a low fluence, the absorption signal decays exponentially. At higher fluences, on the other hand, the decay is more rapid at a shorter time stage but it is almost the same as that at a longer time stage The slower decay is independent of the excitation intensity, suggesting monomolecular decay due to radiative and nonradiative deactivations of singlet excitons. The faster decay is dependent upon the excitation intensity, suggesting higher order kinetics such as bimolecular recombination, most probably due to singlet-singlet exciton annihilation. In other words, the exciton diffusion dynamics can be discussed by analyzing the intensity-dependent faster decay.

The rate equation for the singlet exciton decay is given by (1)

$$
\frac{\mathrm{d} n(t)}{\mathrm{d} t}=-k n(t)-\frac{1}{2} \gamma(t) n^{2}(t)
$$

where $n(t)$ is the exciton density at a delay time $t$ after the laser excitation, $k$ is the monomolecular decay rate due to the 
radiative and non-radiative deactivations, and $\gamma(t)$ is the timedependent bimolecular decay rate due to the singlet-singlet exciton annihilation. The coefficient $1 / 2$ in the second term on the right side represents that one singlet exciton deactivates in the bimolecular annihilation. The monomolecular decay rate $k$ is evaluated as the inverse of the exciton lifetime $\tau$ under weak excitation conditions, which can be measured by the timecorrelated single-photon-counting method. The equation (1) can be simplified by using a new variable (2)

$$
Y(t)=\frac{\exp (-k t)}{n(t)}
$$

By substituting (2) into (1), the differential equation can be solved as

$$
n(t)=\frac{n_{0} \exp (-k t)}{1+\frac{n_{0}}{2} \int_{0}^{t} \gamma(t) \exp (-k t) \mathrm{d} t} .
$$

On the other hand, the time-dependent bimolecular decay rate $\gamma(t)$ is expressed as

$$
\gamma(t)=2 \exp \left(\frac{t}{\tau}\right) \frac{\mathrm{d} Y(t)}{\mathrm{d} t}
$$

Thus, the time dependence of $\gamma(t)$ can be evaluated from the measured values of exciton lifetime $\tau$ and $Y(t)(n(t))$. Here, $n(t)$ is measured by the transient absorption spectroscopy, then is converted to $Y(t)$ by using (2) and $\tau$, and subsequently is converted to $\chi(t)$ by using (4). Fig. 5 shows the $\log -\log$ plots of $\chi(t)$ against time $t$ for three different P3HT films. As shown in the figure, $\chi(t)$ decays with a slope of -0.5 for RR-P3HT crystalline films and is constant independently of time for RRaP3HT amorphous films. These different time dependences can be explained in terms of dimensionality of exciton diffusion as described below.

The diffusion-limited bimolecular rates have been reported to depend on the dimensionality of the system [45]. In the three-dimensional (3D) diffusion, $\chi(t)$ is given by (5)

$$
\gamma_{3 \mathrm{D}}(t)=8 \pi D R\left(1+\frac{R}{\sqrt{2 \pi D t}}\right)
$$

where $D$ is the isotropic diffusion coefficient and $R$ is the effective interaction radius of singlet excitons. For a later time stage $\left(t>>R^{2} /(2 \pi D)\right)$, the annihilation rate can be expressed as the time-independent formula $/ 3 \mathrm{D}=8 \pi D R$. In the twodimensional (2D) diffusion, $\chi(t)$ is given by (6)

$$
\gamma_{2 \mathrm{D}}(t)=\frac{8 D R}{\pi} \int_{0}^{\infty} \frac{\exp \left(-D u^{2} t\right)}{u\left[J_{0}^{2}(u R)+Y_{0}^{2}(u R)\right]^{\mathrm{d} u}}
$$

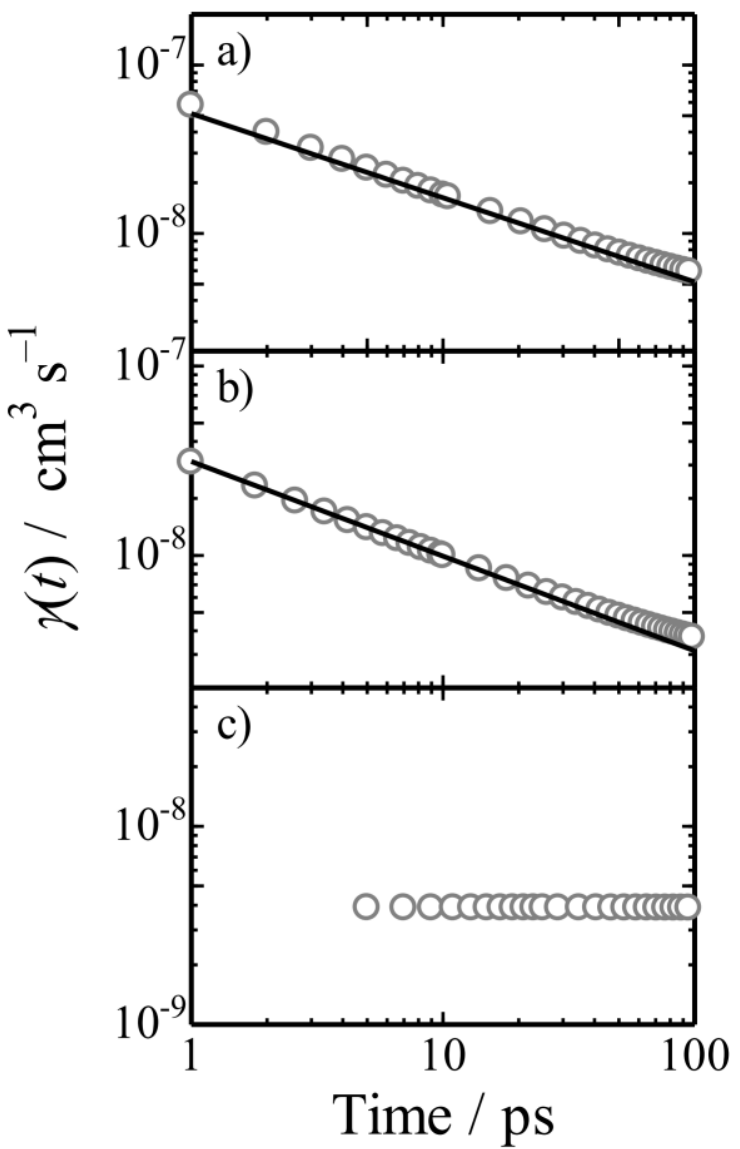

Fig. 5. Time evolution of $\chi(t)$ for P3HT films with different crystallinity: a) RR-P3HT_H and b) RR-P3HT_L excited at $620 \mathrm{~nm}$ and c) RRa-P3HT at 400 $\mathrm{nm}[25]$.

where $J_{0}$ and $Y_{0}$ are the Bessel functions of the first and second kind and zero order, respectively [46]. In the one-dimensional (1D) diffusion, $\chi(t)$ is given by (7)

$$
\gamma_{1 \mathrm{D}}(t)=\frac{1}{R_{a} n_{0}} \sqrt{\frac{8 D}{\pi t}}
$$

In summary, the bimolecular decay rate $\chi(t)$ becomes timeindependent for the 3D diffusion, $t^{-\alpha}(\alpha<0.5)$ dependent for the 2D diffusion at larger $t$, and consistently $t^{-0.5}$ dependent for the 1D diffusion over the whole time domain. Consequently, as shown in Fig. 5, the $t^{-0.5}$ dependent $\gamma(t)$ observed for the RRP3HT films indicates the 1D exciton diffusion and the timeindependent $\chi(t)$ observed for the RRa-P3HT film indicates the $3 \mathrm{D}$ exciton diffusion. These different diffusion dynamics would be due to the different film morphology: RR-P3HT exhibits crystalline films with 1D fibrils [47] and RRa-P3HT exhibits amorphous films.

The diffusion constant $D$ and length $L_{\mathrm{D}}$ can be evaluated from the equations (5) - (7) if the effective interaction radius $R$ is obtained separately, which has been typically assumed to be 1 nm $[42,43]$. Here, we estimate $R$ from the dependence of the initial exciton yield at $0 \mathrm{ps}$ on the excitation intensity. The initial exciton yield is proportional to the excitation intensity under the low excitation conditions, while it is proportional to the square root of the excitation intensity under the high 
excitation conditions. This result indicates bimolecular annihilation above the threshold excitation intensity. Thus, $R$ can be estimated from the initial exciton density at the threshold photon density because exciton diffusion is negligible at $0 \mathrm{ps}$. As summarized in Table 1, $R$ is estimated to be $\sim 3 \mathrm{~nm}$ for P3HT films, and $D$ and $L_{\mathrm{D}}$ increase with increasing crystallinity. The diffusion length $L_{\mathrm{D}}$ in the highly crystalline RR-P3HT_H film is as long as $20 \mathrm{~nm}$, which is 4 times longer than that in the amorphous RRa-P3HT film. This large difference would cause considerable discrepancy in the $L_{\mathrm{D}}$ reported for RR-P3HT crystalline films.

TABLE I

DIFFUSION PARAMETERS OF SINGLET EXCITONS IN P3HT FILMS

\begin{tabular}{lcccc}
\hline \hline \multicolumn{1}{c}{ Polymers } & $R / \mathrm{nm}$ & $D / 10^{-3} \mathrm{~cm}^{2} \mathrm{~s}^{-1}$ & $L_{\mathrm{D}} / \mathrm{nm}$ & Dimension \\
\hline RR-P3HT_H & 3.4 & 7.9 & 20 & $1 \mathrm{D}$ \\
RR-P3HT_L & 3.3 & 3.3 & 14 & 1D \\
RRa-P3HT & 3.2 & 0.46 & 4.8 & $3 \mathrm{D}$ \\
\hline \hline
\end{tabular}

RR-P3HT_H: crystallinity $=64 \%$

RR-P3HT_L: crystallinity $=46 \%$

RRa-P3HT: crystallinity $=0 \%$

The diffusion length $L_{\mathrm{D}}$ is evaluated as $L_{\mathrm{D}}=\sqrt{D \tau}$

\section{B. Singlet Fission}

Spin conversion from singlet to triplet states is generally forbidden because of the transition between different spin multiplicities. Indeed, intersystem crossing from singlet to triplet excitons is spin-forbidden and hence it slowly proceeds on a time scale of nanoseconds in conjugated polymers with a small spin-orbit coupling. On the other hand, singlet fission from one singlet exciton into two triplets is spin-allowed because it is in a four-electron system, which consists of singlet excited and its neighboring ground states at the initial stage, unlike the intersystem crossing in a two-electron system [4850]. Indeed, the singlet fission has been reported to proceed so promptly that it can compete with radiative and non-radiative deactivations of singlet excitons and even with vibrational relaxations in a higher excited state [18,23,51-53]. Therefore, ultrafast transient absorption spectroscopy is a powerful tool for directly detecting prompt triplet generation via singlet fission much more rapid than the intersystem crossing. Note that extreme care should be taken in the assignment of triplet excitons because it is often difficult to distinguish between triplets and polarons from the absorption spectra in the near-IR region alone. An oxygen quenching experiment is useful for the assignment of triplets. However, no oxygen quenching does not always rule out a possibility of triplets because it is not effective for triplets with a short lifetime or with an energy lower than singlet oxygen $(\sim 1 \mathrm{eV})$ [54]. It is highly recommended to measure the transient absorption up to the mid-IR region when the absorption spectra of triplets and polarons are almost the same in the near-IR region.

Singlet fission is a multiple exciton generation process, as mentioned above, and hence has recently attracted much attention because it could potentially improve the photovoltaic efficiency beyond the Shockley-Queisser limit [55-57]. Historically, the singlet fission has been found for molecular crystals such as anthracene crystals [58]. Recent studies on the singlet fission have still focused on molecular acene crystals such as pentacene and rubrene. More than $100 \%$ conversion efficiency has been reported for organic solar cells based on molecular crystals [59]. On the other hand, the singlet fission in conjugated polymer films has also been reported by several groups $[18,23,60-66]$. Here, we describe the singlet fission in two conjugated polymer films with different film morphologies studied by the transient absorption spectroscopy. One is polyfluorene (PFO), which typically forms amorphous films but can form $\beta$-phase films under certain preparation conditions. In the $\beta$ phase, some extended polymer backbones are considered to be aggregated [67]. The other is P3HT, which forms amorphous films for RRa-P3HT and crystalline films for RR-P3HT, as mentioned above.

Fig. 6 shows the transient absorption spectra of PFO amorphous films. The absorption spectrum observed immediately after the laser excitation is safely ascribed to singlet excitons, which exhibits a large peak at $800 \mathrm{~nm}$ and small shoulders at around 600 and $1000 \mathrm{~nm}$. These bands decay in nanoseconds. At $3 \mathrm{~ns}$ after the excitation, an absorption band is observed at $800 \mathrm{~nm}$ alone. This band is ascribed to triplet excitons because it is still observed on a time scale of microseconds and is quenched under an oxygen atmosphere. The time evolution of triplet excitons can be extracted by subtracting the normalized transient absorption signal at 1000 $\mathrm{nm}$ from that at $800 \mathrm{~nm}$. As a result, the decay constant of singlet excitons is estimated to be $11 \mathrm{ps,} \mathrm{which} \mathrm{is} \mathrm{the} \mathrm{same} \mathrm{as}$ the rise constant of triplet excitons. These time constants decrease with increasing excitation intensity, suggesting that triplet generation results from bimolecular singlet-singlet exciton annihilation. As mentioned above, such prompt triplet generation cannot be explained in terms of the spin-forbidden intersystem crossing but rather is ascribable to spin-allowed singlet fission. In other words, prompt triplet generation in amorphous PFO films is due to singlet-singlet exciton annihilation (singlet fusion) followed by singlet fission. Note that triplet generation via the intersystem crossing is negligible because of small spin-orbit coupling. The singlet fission is thermodynamically forbidden from the lowest singlet exciton but allowed from higher singlet excitons generated by singlet-

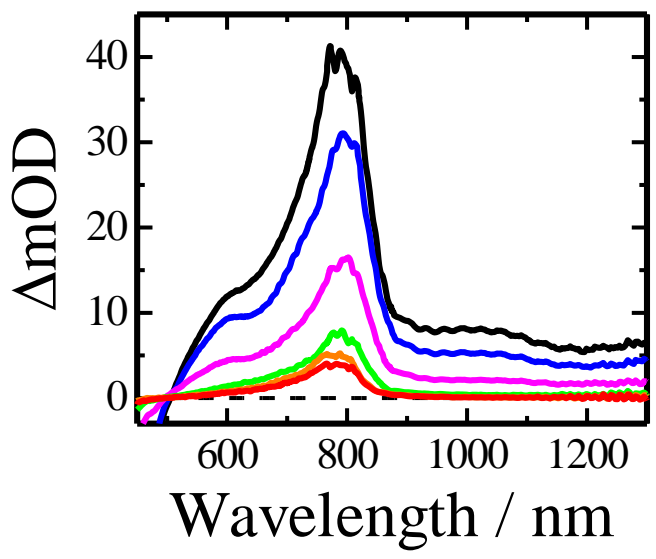

Fig. 6. Transient absorption spectra of PFO amorphous films measured at 0 , $1,10,100,1000$, and $3000 \mathrm{ps}$ after the laser excitation at $400 \mathrm{~nm}$ from top to bottom [23]. 
singlet exciton annihilation, because two triplet pairs generated via the singlet fission is higher in energy than the lowest singlet exciton but lower than higher singlet excitons generated by singlet-singlet exciton annihilation. We therefore conclude that the triplet generation in amorphous PFO films is due to singlet fusion followed by singlet fission.

For $\beta$-phase PFO films, singlet excitons are promptly generated upon the laser excitation and then converted into triplet excitons in tens of picoseconds as is the case of PFO amorphous films. At $3 \mathrm{~ns}$ after the excitation, two absorption bands are observed at 650 and $850 \mathrm{~nm}$, which are ascribed to PFO polarons and triplets, respectively. These two species are generated via singlet-singlet exciton annihilation. In other words, prompt triplet generation in $\beta$-phase PFO films is also due to singlet-singlet exciton annihilation (singlet fusion) followed by singlet fission, which is competitive with polaron generation. Consequently, the triplet generation yield in $\beta$ phase PFO films is reduced to 50\% compared to that in PFO amorphous films. Note that this triplet is due to triplet pairs generated from the singlet fission. The dissociation into free triplets is discussed below.

For PFO amorphous films, triplet excitons decay with a time constant of $1.7 \mathrm{~ns}$, which is too short to be assigned to free triplet excitons. This decay dynamics is independent of the excitation intensity, suggesting monomolecular process. Thus, the triplet decay is ascribed to geminate recombination of triplet pairs to form a singlet exciton (triplet-triplet exciton annihilation). The remaining constant fraction at $6 \mathrm{~ns}$ is ascribed to free triplet excitons dissociated from triplet pairs. For $\beta$-phase PFO films, on the other hand, no triplet decay is observed, indicating that all the triplet pairs are dissociated into free triplet excitons. This is probably because the diffusion constant of triplet excitons is higher in the $\beta$ phase than in amorphous domains.

In summary, singlet fusion followed by singlet fission is observed both for amorphous and $\beta$-phase PFO films. The formation yield of triplet pairs is reduced to $50 \%$ in $\beta$-phase PFO films compared in amorphous PFO films. This is because polarons are competitively generated in $\beta$-phase PFO films. The dissociation efficiency of triplet pairs is $100 \%$ for $\beta$-phase PFO films but $50 \%$ for amorphous PFO films. The lower dissociation efficiency is probably because smaller diffusion constant of triplet excitons in amorphous domains. As a result, there is not much difference in the overall singlet fission efficiency between amorphous and $\beta$-phase PFO films. For RRa-P3HT amorphous films, a similar singlet fusion followed by singlet fission is observed. For crystalline RR-P3HT films, on the other hand, no singlet fission is observed even though it is energetically as possible as in RRa-P3HT films. Instead, polarons are generated from higher singlet excitons formed by singlet-singlet exciton annihilation. In highly crystalline RRP3HT films, there are crystalline and amorphous domains with different ionization potentials, which would promote polaron generation even in RR-P3HT neat films [68].

In conclusion, disordered amorphous domains are beneficial for efficient triplet pair generation but suffer from low dissociation efficiency of triplet pairs to free triplets. On the other hand, ordered domains such as $\beta$ phase or crystalline phase are beneficial for efficient dissociation of triplet pairs but are likely to cause other competitive processes such as polaron generation from higher singlet excitons. We therefore speculate that the singlet fission in crystalline polymers would be efficient from an exciton as low in energy as possible.

\section{Charge Dynamics}

We have studied the charge generation and geminate recombination dynamics in a series of polymer/fullerene blend films as shown in Fig. 7 by femtosecond transient absorption spectroscopy. Here, we focus on RRa-P3HT/PCBM as a representative amorphous blend film and RR-P3HT/PCBM as a representative crystalline blend film. Later, we will summarize the charge generation and recombination dynamics in terms of crystallinity of conjugated polymers. We further describe the bimolecular recombination dynamics in RRP3HT/PCBM blends by microsecond transient absorption spectroscopy. The geminate recombination cannot be distinguished from the bimolecular recombination in terms of the absorption spectra, because polymer polaron and PCBM anion should be observed in either recombination dynamics. The geminate recombination is monomolecular decay process and hence is independent of the excitation intensity. On the other hand, the bimolecular recombination is bimolecular decay process and hence is dependent on the excitation intensity. Thus, one can distinguish between geminate and bimolecular recombination by analyzing the dependence of the decay dynamics on the excitation intensity.
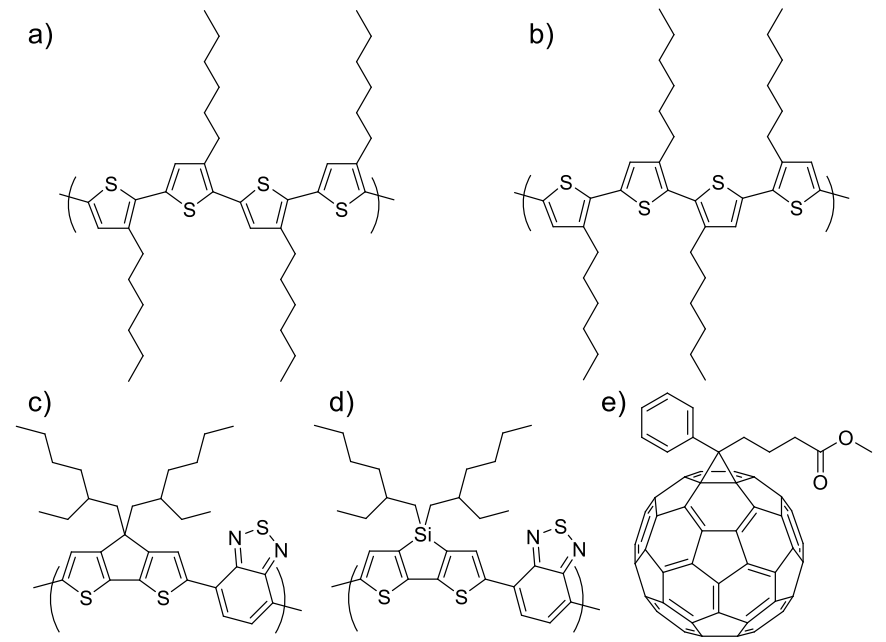

Fig. 7. Chemical structures of conjugated donor polymers with different crystallinity and an acceptor fullerene: a) RR-P3HT, b) RRa-P3HT, c) PCPDTBT, d) PSBTBT, and e) PCBM.

\section{A. Charge Generation and Geminate Recombination}

In amorphous or less crystalline polymers such as RRa-P3HT blended with PCBM, singlet excitons rapidly disappear in a few picoseconds after the laser excitation. At the same time, polymer polarons are promptly generated. For RRaP3HT/PCBM blend films, as shown in Fig. 8, the singlet exciton band is reduced by half even at 0 ps and completely 


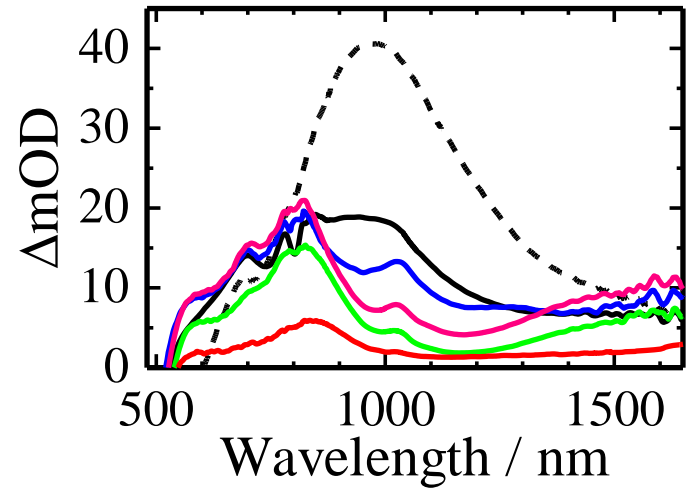

Fig. 8. Transient absorption spectra of RRa-P3HT/PCBM amorphous films measured at $0,0.2,1,100$, and $3000 \mathrm{ps}$ after the laser excitation at $400 \mathrm{~nm}$ from top to bottom. The broken line represents the transient absorption spectrum at 0 ps of RRa-P3HT neat films [19].

disappears in a picosecond. This efficient exciton quenching is consistent with almost 100\% PL quenching of the blend. A similar prompt polaron generation has also been found for low or modest crystalline polymer blends such as PCPDTBT/PCBM and PSBTBT/PCBM. The prompt polaron generation in a picosecond is indicative of no contribution of the exciton diffusion to the charge generation. These findings suggest that polymer and PCBM are well mixed on a molecular scale and that polymer crystalline domains are comparable to exciton delocalization size.

In highly crystalline RR-P3HT blended with PCBM, as shown in Fig. 9, the singlet exciton band is reduced by half even at $0 \mathrm{ps}$, suggesting prompt polaron generation similar to RRaP3HT/PCBM blends. Interestingly, the singlet exciton band decays slowly in tens of picoseconds. This time constant is dependent on the blend ratio or annealing conditions: it increases with increasing P3HT domain size. Thus, the delayed polaron generation is ascribed to charge generation following after the exciton diffusion to a polymer/fullerene interface in large P3HT crystalline domains. The domain size is roughly estimated to be in the order of $\sim 10 \mathrm{~nm}$ by using a diffusion constant of $8 \times 10^{-3} \mathrm{~cm}^{2} \mathrm{~s}^{-1}$ and a rise time of $30 \mathrm{ps}$, which is consistent with fibril structures observed by TEM images $[69,70]$. In summary, there are two charge generation pathways in highly crystalline RR-P3HT blended with PCBM. One is the prompt charge generation at the $\mathrm{P} 3 \mathrm{HT} / \mathrm{PCBM}$ interface and the other is the delayed charge generation following after the exciton diffusion to the interface in large crystalline domains.

At a later time stage up to nanoseconds, charge

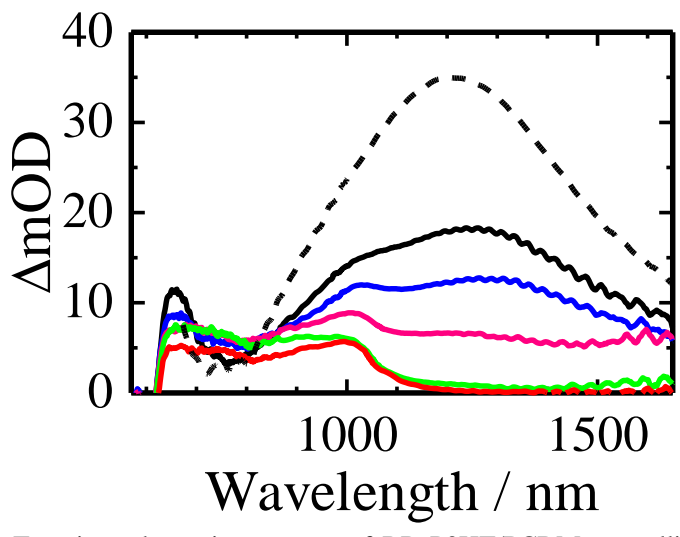

Fig. 9. Transient absorption spectra of RR-P3HT/PCBM crystalline films measured at $0,1,10,100$, and $3000 \mathrm{ps}$ after the laser excitation at $400 \mathrm{~nm}$ from top to bottom. The broken line represents the transient absorption spectrum at 0 ps of RR-P3HT neat films [19].

recombination dynamics can be observed. For amorphous or less crystalline polymers such as RRa-P3HT blended with PCBM, polymer polarons decay in a nanosecond. This decay dynamics is independent of the excitation intensity and is in good agreement with the recovery dynamics of the photobleaching in the ground state, suggesting that polymer polaron and PCBM anion pairs geminately recombine to the ground state. Furthermore, the decay constant is in good agreement with the lifetime of the charge transfer (CT) emission of this blend measured by the time-correlated singlephoton-counting method. We therefore ascribe the decay dynamics to the geminate recombination of interfacial CT state that is formed by polymer polaron and PCBM anion pairs. A part of polarons remain at a few nanoseconds after the excitation, and are still observed on a time scale of microseconds. These long-lived polarons are ascribed to dissociated charge carriers. The dissociation efficiency is as low as 30\% for RRa-P3HT/PCBM, while it is as high as $>90 \%$ for RR-P3HT/PCBM blends. As summarized in Table 2, there is a good correlation between the charge dissociation efficiency and the crystallinity of donor polymers. We will describe this correlation later.

\section{B. Bimolecular Recombination}

Here, we describe the bimolecular recombination dynamics in RR-P3HT/PCBM blend films on a time scale of microseconds. As shown in Fig. 10, two absorption bands are observed at around 700 and $1000 \mathrm{~nm}$. These bands are not quenched under oxygen atmosphere and hence ascribed to

TABLE II

PHOTOVOLTAIC CONVERSION EFFICIENCY OF POLYMER/PCBM SOLAR CELLS

\begin{tabular}{|c|c|c|c|c|c|c|}
\hline Polymers & Phase & $\eta_{\mathrm{ED}}$ & $\eta_{\mathrm{CT}}$ & $\eta_{\mathrm{CD}}$ & $L_{010} / \mathrm{nm}$ & $d / \AA$ \\
\hline RR-P3HT with annealing & Highly crystalline & 0.91 & 1 & 0.93 & 12 & 3.8 \\
\hline RR-P3HT without annealing & Modest crystalline & 0.95 & 1 & 0.8 & 5.7 & 3.8 \\
\hline PSBTBT & Less crystalline & 1 & 1 & 0.75 & 4.6 & 3.5 \\
\hline PCPDTBT with additive & Slightly ordered & 1 & 1 & 0.7 & $(1.1)$ & $(3.8)$ \\
\hline PCPDTBT without additive & Less ordered & 1 & 1 & 0.5 & $(0.5)$ & (3.9) \\
\hline RRa-P3HT & Amorphous & 1 & 1 & 0.3 & & \\
\hline
\end{tabular}

Photovoltaic conversion efficiencies are cited from Refs 19, 22, 28, and 71.

Crystallographic data are taken from Refs $72-76$. The values in parentheses are data for polymer neat films. 
$>$ REPLACE THS LINE WITH YOUR PAPER IDENTIFICATION NUMBER (DOUBLE-CLICK HERE TO EDIT)

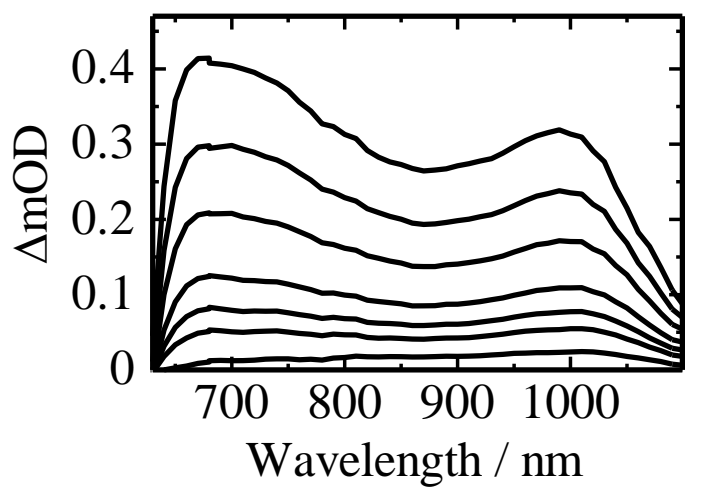

Fig. 10. Transient absorption spectra of RR-P3HT/PCBM blend films measured at $0.5,1,2,5$, and $10 \mu$ s after the laser excitation at $400 \mathrm{~nm}[30]$.

polarons. Interestingly, the band at $700 \mathrm{~nm}$ decays faster than that at $1000 \mathrm{~nm}$, suggesting that there exist two different polarons in the blend. The decay dynamics is dependent on the excitation intensity and is followed by a power law equation (8)

$$
n(t)=\frac{n_{0}}{(1+a t)^{\alpha}}
$$

Such power law equation has been theoretically derived in terms of energetic disorders in polymer films. [77,78] The slope $\alpha$ is estimated to be unity for the band at $700 \mathrm{~nm}$ and 0.5 for the band at $1000 \mathrm{~nm}$, suggesting bimolecular recombination of trap-free and trapped polarons, respectively.

The rate equation of diffusion-limited bimolecular recombination is given by (9)

$$
\frac{\mathrm{d} n(t)}{\mathrm{d} t}=-\gamma(t) n^{2}(t)
$$

where $n(t)$ is the carrier density and $\chi(t)$ is the bimolecular recombination rate at a time $t$. By substituting (8) into (9), the time dependent $\gamma(t)$ is obtained as (10)

$$
\gamma(t)=\frac{a \alpha}{n_{0}}(1+a t)^{\alpha-1}
$$

By substituting (8) into (10), the density dependent $\chi(n)$ is obtained as (11)

$$
\gamma(n)=\frac{a \alpha}{n}\left(\frac{n}{n_{0}}\right)^{\frac{1}{\alpha}}
$$

Thus, the carrier lifetime $\tau_{0}$ at a carrier density of $n_{0}$ is given by (12)

$$
\tau_{0}=\frac{1}{\gamma_{0} n_{0}}=\frac{1}{a \alpha}
$$

By using the kinetic parameters of $a$ and $\alpha$, the bimolecular recombination rate can be obtained. As shown in Fig. 11, it is


Fig. 11. a) Time-dependent bimolecular recombination rate $\chi(t)$ of the polaron band at $700 \mathrm{~nm}$ (solid line) and at $1000 \mathrm{~nm}$ (broken line). b) Carrier-densitydependent bimolecular recombination rate $\chi(n)$ of the polaron band at $700 \mathrm{~nm}$ (solid line) and at $1000 \mathrm{~nm}$ (broken line) [30].

independent of time or carrier density for the band at $700 \mathrm{~nm}$ and decreases with time and carrier density for the band at 1000 $\mathrm{nm}$. The effective activation energy can be evaluated from the temperature dependence of $\gamma(t)$. For the polaron band at 700 $\mathrm{nm}$, the activation energy $E_{\mathrm{A}}$ is independent of the carrier density, and estimated to be $0.078 \mathrm{eV}$. For the polaron band at $1000 \mathrm{~nm}$, on the other hand, the $E_{\mathrm{A}}$ increases with decreasing carrier density, and varies from 0.097 to $0.178 \mathrm{eV}$ over the carrier density range from $10^{17}$ to $10^{16} \mathrm{~cm}^{-3}$. We therefore ascribe the band at $700 \mathrm{~nm}$ to trap-free polarons and the band at $1000 \mathrm{~nm}$ to trapped polarons.

\section{Relevance to Photovoltaic EfFiciency}

\section{A. Efficient Charge Generation}

The charge dissociation efficiency has critical impact on the photovoltaic performance because it becomes the difference between free charge carrier generation and charge recombination loss. As mentioned above, it can be quantitatively evaluated by the transient absorption spectroscopy. Table 2 summarizes each photovoltaic conversion efficiency in a series of polymer/fullerene blends with different crystallinity. The exciton diffusion efficiency $\eta_{\mathrm{ED}}$ is unity for amorphous or less crystalline polymers except for highly crystalline RR-P3HT. In such blends with high $\eta_{\mathrm{ED}}$, the charge generation is completed in a picosecond, suggesting that almost all the excitons encounter with surrounding PCBM molecules without exciton diffusion. These finding indicate that, as shown in Fig. 12, polymer and PCBM are intimately 
mixed in the amorphous phase and that the domain size of less crystalline polymers is not large but rather comparable to the exciton delocalization size. On the other hand, as shown in the figure, the domain size of highly crystalline RR-P3HT is so large that excitons should diffuse to a polymer/fullerene interface to convert to charge carriers. As a result, $10 \%$ of the excitons are lost before arriving at the interface. The charge transfer efficiency $\eta_{\mathrm{CT}}$ is estimated to be unity for all the blend films because such prompt charge generation in a picosecond can compete kinetically with the other loss processes such as geminate recombination and radiative and non-radiative deactivations. Most interestingly, the charge dissociation efficiency $\eta_{\mathrm{CD}}$ is dependent upon the crystallinity of polymers. The $\eta_{\mathrm{CD}}$ is as low as $30 \%$ for RRa-P3HT/PCBM amorphous blend films, increases with increasing crystallinity, and is as high as $>90 \%$ for RR-P3HT/PCBM highly crystalline blend films. This correlation indicates that crystalline polymer domains have critical impact on the charge dissociation in polymer solar cells.

The crystalline polymer domains would be beneficial for the efficient charge dissociation for the following reasons as discussed previously [19,28]. First, the crystalline polymer domains would induce charge delocalization and hence extend the effective distance between electron and hole pairs, resulting in reduced Coulomb interaction. Recently, Deibel et al. have demonstrated that the charge delocalization of polymer polarons can enhance the $\eta_{\mathrm{CD}}$ effectively by kinetic Monte Carlo simulation [79]. As shown in Table 2, the $\eta_{\mathrm{CD}}$ is not correlated with the $\pi-\pi$ stacking distance $d$, but rather correlated with the crystalline size in the $\pi$ stacking direction $L_{010}$, suggesting that the charge delocalization would be more important in the $\pi$ stacking direction. More interestingly, the $\eta_{\mathrm{CD}}$ is highly efficient for crystalline polymers with a correlation length of $L_{010}>4 \mathrm{~nm}$. This is comparable to the effective Coulomb capture radius estimated by taking into account the entropy gain associated with changing from a single exciton to two separated charges as suggested by Durrant et al. [8]. Second, the polymer crystallization would cause higher local hole mobility, which also can improve the $\eta_{\mathrm{CD}}$ effectively. Indeed, the local hole mobility of RR-P3HT has been reported to be as high as $0.1-30 \mathrm{~cm}^{2} \mathrm{~V}^{-1} \mathrm{~s}^{-1}$ by time-resolved $\mathrm{THz}$ spectroscopy [80-82]. Recently, McGehee et al. have demonstrated that highly efficient charge dissociation can be explained by using a locally high charge mobility and an energetic cascade in a three-phase bulk heterojunction in polymer/fullerene solar cells [82]. In such a three-phase bulk heterojunction, hole polarons would transfer from disordered amorphous phase mixed with PCBM to more stable ordered crystalline phase. We have found such hole transfer for crystalline polymer/fullerene blends such as RR-P3HT/PCBM and PSBTBT/PCBM [19,28]. Third, the polymer crystallization would expel PCBM molecules from crystalline to amorphous domains, resulting in formation of PCBM clusters. Recent studies have suggested that PCBM clusters also have critical impact on the charge dissociation [83-86]. As is the case with the hole delocalization, PCBM clusters would a)



b)



c)


Fig. 12. Blend morphology a) in amorphous polymer films blended with PCBM like RRa-P3HT/PCBM and b) in crystalline polymer films blended with PCBM like RR-P3HT/PCBM. c) There are three characteristic phases in crystalline polymer/fullerene blends: crystalline polymer domains, disordered polymer domains mixed with PCBM molecules, and PCBM aggregate domains.

induce electron delocalization and hence extend the effective distance between electron and hole pairs, resulting in reduced Coulomb interaction. Similarly to the hole transfer from disordered to crystalline polymer domains, electrons would transfer from PCBM dispersed in disordered polymer domains to more stable aggregated PCBM cluster domains [83].

\section{B. Long-Lived Charge Carriers}

The dissociated charge carriers should be collected to each electrode before recombining bimolecularly for efficient photovoltaic performance. In other words, the charge carrier lifetime should be longer than the charge collection time. For RR-P3HT/PCBM blend films, as mentioned before, the bimolecular recombination rate is estimated to be less than $\sim 10^{-12} \mathrm{~cm}^{3} \mathrm{~s}^{-1}$ from the power-law decay analysis for transient absorption measurements under open-circuit conditions. On the other hand, the Langevin recombination rate is given by $\gamma_{\mathrm{L}}$ $=q \mu / \varepsilon \varepsilon_{0}$ where $\varepsilon$ is the dielectric constant of the active layer, $\varepsilon_{0}$ is the vacuum permittivity, and $q$ is the elementary charge. Assuming $\mu=10^{-4} \mathrm{~cm}^{2} \mathrm{~V}^{-1} \mathrm{~s}^{-1}$, $\gamma$ is estimated to be $\sim 10^{-10} \mathrm{~cm}^{2}$ $\mathrm{V}^{-1} \mathrm{~s}^{-1}$. This is more than two orders of magnitude faster than the observed one. On the other hand, the carrier lifetime $\tau_{0}$ is evaluated to be longer than $10 \mu \mathrm{s}$ at a carrier density of $10^{16}$ $10^{17} \mathrm{~cm}^{-3}$, which is a typical carrier density under open-circuit condition at the 1 sun illumination. Note that the $\tau_{0}$ is not obtained at a steady state like device operation conditions. More correctly, therefore, it is necessary to evaluate the carrier lifetime by quasi-steady-state methods such as transient photovoltage (TPV) and photocurrent (TPC) measurements. For RR-P3HT/PCBM, the $\tau_{0}$ is in good agreement with that 
evaluated by the TPV/TPC measurements [87]. Under the short-circuit condition, on the other hand, the charge collection time $\tau_{\mathrm{CC}}$ is roughly estimated as a transit time: $\tau_{\mathrm{CC}}=L^{2} / 2 \mu V_{\mathrm{b}}$ where $L$ is the active layer thickness, $\mu$ is the carrier mobility, and $V_{\mathrm{b}}$ is the build-in potential in the active layer. The carrier density is estimated by $n_{\mathrm{SC}}=J_{\mathrm{SC}} \tau_{\mathrm{CC}} / q L \eta_{\mathrm{CD}}$. For typical cases, $\tau_{\mathrm{CC}}$ and $n_{\mathrm{SC}}$ are estimated to be $<1 \mu$ s and $10^{15} \mathrm{~cm}^{-3}$, respectively, assuming that $J_{\mathrm{SC}}=10 \mathrm{~mA} \mathrm{~cm}{ }^{-2}, L=100 \mathrm{~nm}, \mu=10^{-4} \mathrm{~cm}^{2} \mathrm{~V}^{-1}$ $\mathrm{s}^{-1}$, and $V_{\mathrm{b}} \approx V_{\mathrm{OC}} \approx 1 \mathrm{~V}$. For RR-P3HT/PCBM blends, therefore, the carrier lifetime $\tau_{\mathrm{SC}}$ is estimated to be $>10 \mu \mathrm{s}$, which is at least one order of magnitude longer than the charge collection time $\tau_{\mathrm{CC}}$. This is the origin of highly efficient charge collection in RR-P3HT/PCBM solar cells even with a thick active layer $(\sim 200 \mathrm{~nm})$. If the bimolecular recombination obeys the Langevin recombination, the carrier lifetime $\tau_{\mathrm{L}}$ is estimated be $<1 \mu$ s, which is comparable to the charge collection time. In other words, the efficient charge collection in RR-P3HT/PCBM blends originates from the reduced recombination.

The long-lived charge carriers due to the reduced recombination dynamics is the key to the efficient charge collection. Currently, several explanations have been proposed $[16,88,89]$. One possible explanation is that the recombination is not diffusion-limited because of highly efficient charge dissociation as described above.[22] For such non-diffusionlimited recombination, the apparent recombination rate $\gamma_{\mathrm{a}}$ is given by (13)

$$
\gamma_{\mathrm{a}}=\frac{k_{\mathrm{rec}}}{k_{\mathrm{dis}}+k_{\mathrm{rec}}} \gamma_{\mathrm{L}}=\left(1-\eta_{\mathrm{cD}}\right) \gamma_{\mathrm{L}}
$$

where $k_{\text {rec }}$ is the recombination rate and $k_{\text {dis }}$ is the dissociation rate. For RR-P3HT/PCBM blends, as mentioned before, the $\eta_{\mathrm{CD}}$ is as high as $>0.95$ and hence the $\gamma_{\mathrm{a}}$ would be reduced by a factor of $<0.05$. In crystalline domains, polymer polarons are promptly generated within a laser pulse duration $(<100 \mathrm{fs})$ and the geminate recombination is negligible up to a few nanoseconds. In other words, the dissociation rate $k_{\text {dis }}$ is as fast as $>(100 \mathrm{fs})^{-1} \approx 10^{13} \mathrm{~s}^{-1}$ while the recombination rate $k_{\text {rec }}$ is as slow as $\left\langle(1 \mathrm{~ns})^{-1} \approx 10^{9} \mathrm{~s}^{-1}\right.$. Thus, the $\gamma_{\mathrm{a}}$ would be reduced by a factor of $<10^{-4}$. We note that this model is based on an assumption that the CT state photogenerated is the same as that generated via the charge recombination although the former CT state might be energetically higher than the latter CT state. In addition, the three-phase bulk heterojunction structure mentioned above would suppress the charge recombination effectively because electrons and holes are energetically likely to remain at the P3HT crystalline phase and the PCBM aggregate phase, respectively, rather than in disordered mixed phases [90]. Further study is needed to solve this important issue.

\section{REFERENCES}

[1] R. G. W. Norrish and G. Porter, "Chemical reactions produced by very high light intensities," Nature, vol. 164, p. 658, Oct. 1949.

[2] G. Porter, "Flash photolysis and spectroscopy. A new method for the study of free radical reactions," Proc. Roy. Soc. London, Ser. A, vol. 200, no. 1061, pp. 284-300, Jan. 1950.
[3] R. G. W. Norrish, "Some fast reactions in gases studied by flash photolysis and kinetic spectroscopy," Nobel Lecture, [Online] Available: http://www.nobelprize.org/nobel_prizes/chemistry/laureates/1967/norris h-lecture.html

[4] G. Porter, "Flash photolysis and some of its applications," Nobel Lecture, [Online] Available: http://www.nobelprize.org/nobel_prizes/chemistry/l aureates/1967/porter-lecture.html

[5] A. Zewail, "Femtochemistry: Atomic-scale dynamics of the chemical bond using ultrafast lasers," Nobel Lecture, [Online] Available: http://www.nobelprize.org/nobel_prizes/chemistry/laureates/1999/zewail -lecture.html

[6] B. Kippelen and J.-L. Brédas, "Organic photovoltaics," Energy Environ. Sci., vol. 2, no. 3, pp. 251-261, Mar. 2009.

[7] C. Deibel and V. Dyakonov, "Polymer-fullerene bulk heterojunction solar cells," Rep. Prog. Phys., vol. 73, no. 9, pp. 096401-1-096401-39, Sept. 2010.

[8] T. M. Clarke and J. R. Durrant, "Charge photogeneration in organic solar cells," Chem. Rev., vol. 110, no. 11, pp. 6736-6767, Nov. 2010.

[9] H. Ohkita and S. Ito, "Transient absorption spectroscopy of polymerbased thin-film solar cells," Polymer, vol. 52, no. 20, pp. 4397-4417, Sept. 2011.

[10] H. Ohkita and S. Ito, "Exciton and charge dynamics in polymer solar cells," in Organic Solar Cells, W. C. H. Choy, Ed. London: Springer-Verlag, 2013, pp. 103-137.

[11] G. Li, R. Zhu, and Y. Yang, "Polymer solar cells," Nat. Photon., vol. 6, no. 3, pp. 153-161, Mar. 2012

[12] S. R. Cowan, N. Banerji, W. L. Leong, and A. J. Heeger, "Charge formation, recombination, and sweep-out dynamics in organic solar cells," Adv. Funct. Mater., vol. 22, no. 6, pp. 1116-1128, Mar. 2012.

[13] C. M. Proctor, M. Kuik, and T.-Q. Nguyen, "Charge carrier recombination in organic solar cells," Prog. Polym. Sci., vol. 38, no. 12, pp. 1941-1960, Dec. 2013

[14] L. Dou, J. You, Z. Hong, Z. Xu, G. Li, R. A. Street, and Y. Yang, "25th anniversary article: A decade of organic/polymeric photovoltaic research," Adv. Mater., vol. 25, no. 46, pp. 6642-6671, Dec. 2013.

[15] S. D. Dimitrov and J. R. Durrant, "Materials design considerations for charge generation in organic solar cells," Chem. Mater., vol. 26, no. 1, pp. 616-630, Jan. 2014

[16] G. Lakhwani, A. Rao, and R. H. Friend, "Bimolecular recombination in organic photovoltaics," Annu. Rev. Phys. Chem., vol. 65, pp. 557-581, Apr. 2014.

[17] F. Gao and O. Inganäs, "Charge generation in polymer-fullerene bulkheterojunction solar cells," Phys. Chem. Chem. Phys., vol. 16, no. 38, pp. 20291-20304, Oct. 2014

[18] J. Guo, H. Ohkita, H. Benten, and S. Ito, "Near-IR femtosecond transient absorption spectroscopy of ultrafast polaron and triplet formation in polythiophene films with different regioregularities." J. Am. Soc. Chem., vol. 131, no. 46, pp. 16869-16880, Nov. 2009.

[19] J. Guo, H. Ohkita, H. Benten, and S. Ito, "Charge generation and recombination dynamics in poly(3-hexylthiophene)/fullerene blend films with different regioregularities and morphologies." J. Am. Soc. Chem., vol. 132, no. 17, pp. 6154-6164, May 2010.

[20] H. Ohkita, J. Kosaka, J. Guo, H. Benten, and S. Ito, "Charge generation dynamics in polymer/polymer solar cells studied by transient absorption spectroscopy," J. Photon. Energy, vol. 1, no. 1, pp. 011118-1-011118-12, Mar. 2011.

[21] S. Honda, S. Yokoya, H. Ohkita, H. Benten, and S. Ito, "Light-harvesting mechanism in polymer/fullerene/dye ternary blend studied by transient absorption spectroscopy." J. Phys. Chem. C, vol. 115, no. 22, pp. 1130611317, June 2011.

[22] S. Yamamoto, H. Ohkita, H. Benten, and S. Ito, "Role of interfacial charge transfer state in charge generation and recombination in low-bandgap polymer solar cell," J. Phys. Chem. C, vol. 116, no. 28, pp. 14804-14810, July 2012.

[23] Y. Tamai, H. Ohkita, H. Benten, and S. Ito, "Singlet fission in poly $(9,9$ 'di-n-octylfluorene films," J. Phys. Chem. C, vol. 117, no. 20, pp. $10277-$ 10284, May 2013

[24] S. Yamamoto et al., "Efficient charge generation and collection in amorphous polymer solar cells," J. Phys. Chem. C, vol. 117, no. 22, pp. 11514-11521, June 2013

[25] Y. Tamai, Y. Matsuura, H. Ohkita, H. Benten, and S. Ito, "Onedimensional singlet exciton diffusion in poly(3-hexylthiophene) crystalline domains," J. Phys. Chem. Lett., vol. 5, no. 2, pp. 399-403, Jan. 2014 
[26] Y. Tamai, H. Ohkita, H. Benten, and S. Ito, "Triplet exciton dynamics in fluorene-based copolymers with different aromatic amines," Chem. Mater., vol. 26, no. 8, pp. 2733-2742, Apr. 2014.

[27] S. Yamamoto et al., "Charge generation and recombination in fullereneattached poly(3-hexylthiophene)-based diblock copolymer films," J. Phys. Chem. C, vol. 118, no. 20, pp. 10584-10589, May 2014.

[28] Y. Tamai, K. Tsuda, H. Ohkita, H. Benten, and S. Ito, "Charge-carrier generation in organic solar cells using crystalline donor polymers," Phys. Chem. Chem. Phys., vol. 16, no. 38, pp. 20338-20346, Oct. 2014.

[29] S. Yamamoto, J. Guo, H. Ohkita, and S. Ito, "Formation of methanofullerene cation in bulk heterojunction polymer solar cells studied by transient absorption spectroscopy," Adv. Funct. Mater., vol. 18 , no. 17, pp. 2555-2562, Sept. 2008.

[30] J. Guo, H. Ohkita, H. Benten, and S. Ito, "Bimodal polarons and hole transport in poly(3-hexylthiophene):fullerene blend films." J. Am. Soc. Chem., vol. 132, no. 28, pp. 9631-9637, Aug. 2010.

[31] S. Yamamoto, H. Ohkita, H. Benten, and S. Ito, "Formation mechanism of fullerene cation in bulk heterojunction polymer solar cells," Adv. Funct. Mater., vol. 22, no. 14, pp. 3075-3082, July 2012.

[32] D. E. Markov, J. C. Hummelen, and P. W. M. Blom, "Dynamics of exciton diffusion in poly( $p$-phenylene vinylene)/fullerene heterostructures," Phys. Rev. B, vol. 72, no. 4, pp. 045216-1-045216-5, July 2005.

[33] D. E. Markov and P. W. M. Blom, "Migration-assisted energy transfer at conjugated polymer/metal interfaces," Phys. Rev. B, vol. 72, no. 16, pp. 161401-1-161401-4, Oct. 2005.

[34] D. E. Markov and P. W. M. Blom, "Anisotropy of exciton migration $\mathrm{n}$ poly(p-phenylene vinylene)," Phys. Rev. B, vol. 74, no. 8, pp. 085206-1085206-1-085206-5, Aug. 2006.

[35] S. R. Scully and M. D. McGehee, "Effects of optical interference and energy transfer on exciton diffusion length measurements in organic semiconductors," J. Appl. Phys., vol. 100, no. 3, pp. 034907-1-034907-5, Aug. 2006

[36] A. J. Lewis, A. Ruseckas, O. P. M. Gaudin, G. R. Webster, P. L. Burn, and I. D. W. Samuel, "Singlet exciton diffusion in MEH-PPV films studied by exciton-exciton annihilation," Org. Electron., vol. 7, no. 6, pp. 452-456, Dec. 2006.

[37] O. V. Mikhnenko, F. Cordella, A. B. Sieval, J. C. Hummelen, P. W. M. Blom, and M. A. Loi, "Temperature dependence of exciton diffusion in conjugated polymers," J. Phys. Chem. B, vol. 112, no. 37, pp. 1160111604, Sept. 2008.

[38] J. E. Kroeze, T. J. Savenije, M. J. W. Vermeulen, and J. M. Warman, "Contactless determination of the photoconductivity action spectrum, exciton diffusion length, and charge separation efficiency in polythiophene-sensitized $\mathrm{TiO}_{2}$ bilayers," J. Phys. Chem. B, vol. 107, no. 31, pp. 7696-7705, Aug. 2003.

[39] L. Lüer et al., "Oxygen-induced quenching of photoexcited states in polythiophene films," Org. Electron., vol. 5, no. 1-3, pp. 83-89, Mar. 2004

[40] C. Goh, S. R. Scully, and M. D. McGehee, "Effects of molecular interface modification in hybrid organic-inorganic photovoltaic cells," J. Appl. Phys., vol. 101, no. 11, pp. 114503-1-114503-12, June 2007.

[41] A. Huijser, T. J. Savenije, A. Shalav, and L. D. A. Siebbeles, "An experimental study on the molecular organization and exciton diffusion in a bilayer of a porphyrin and poly(3-hexylthiophene)," J. Appl. Phys., vol. 104, no. 3, pp. 034505-1-034505-10, Aug. 2008.

[42] P. E. Shaw, A. Ruseckas, and I. D. W. Samuel, "Exciton diffusion measurements in poly(3-hexylthiophene)," Adv. Mater., vol. 20, no. 18, pp. 3516-3520, Sept. 2008.

[43] S. Cook, H. Liyuan, A. Furube, and R. Katoh, "Singlet annihilation in films of regioregular poly(3-hexylthiophene): Estimates for singlet diffusion lengths and the correlation between singlet annihilation rates and spectral relaxation," J. Phys. Chem. C, vol. 114, no. 24, pp. 1096210968, July 2010.

[44] C. Leow, T. Ohnishi, and M. Matsumura, "Diffusion lengths of excitons in polymers in relation to external quantum efficiency of the photocurrent of solar cells," J. Phys. Chem. C, vol. 118, no. 1, pp. 71-76, Jan. 2014.

[45] E. Engel, K. Leo, and M. Hoffmann, "Ultrafast relaxation and excitonexciton annihilation in PTCDA thin films at high excitation densities," Chem. Phys., vol. 325, no. 1, pp. 170-177, June 2006.

[46] K. R. Naqvi, "Diffusion-controlled reactions in two-dimensional fluids: Discussion of measurements of lateral diffusion of lipids in biological membranes," Chem. Phys. Lett., vol. 28, no. 2, pp. 280-284, Sept. 1974.
[47] J. A. Lim, F. Liu, S. Ferdous, M. Muthukumar, and A. L. Briseno, "Polymer semiconductor crystals," Mater. Today, vol. 13, no. 5, pp. 1424, May 2010.

[48] M. B. Smith and J. Michl, "Singlet fission," Chem. Rev., vol. 110, no. 11, pp. 6891-6936, Nov. 2010.

[49] M. B. Smith and J. Michl, "Recent advances in singlet fission," Annu. Rev. Phys. Chem., vol. 64, pp. 361-386, Apr. 2013.

[50] G. B. Piland, J. J. Burdett, R. J. Dillon, and C. J. Bardeen, "Singlet fission: From coherences to kinetics," J. Phys. Chem. Lett., vol. 5, no. 13, pp. 2312-2319, July 2014.

[51] R. Katoh and M. Kotani, "Fission of a higher excited state generated by singlet exciton fusion in an anthracene crystal," Chem. Phys. Lett., vol. 196, no. 1-2, pp. 108-112, Aug. 1992.

[52] R. Katoh, M. Kotani, Y. Hirata, and T. Okada, "Triplet exciton formation in a benzophenone single crystal studied by picosecond time-resolved absorption spectroscopy," Chem. Phys. Lett., vol. 264, no. 6, pp. 631-635, Jan. 1997.

[53] S. Watanabe, A. Furube, and R. Katoh, "Generation and decay dynamics of triplet excitons in $\mathrm{Alq}_{3}$ thin films under high-density excitation conditions," J. Phys. Chem. A, vol. 110, no. 34, pp. 10173-10178, Aug. 2006.

[54] N. J. Turro, "Energy transfer" in Modern Molecular Photochemistry, 2nd ed. Mill Valley, CA, USA: Univ. Science Books, 1991, ch. 9, sec. 21, p. 354

[55] M. C. Hanna and A. J. Nozik, "Solar conversion efficiency of photovoltaic and photoelectrolysis cells with carrier multiplication absorbers," J. Appl. Phys., vol. 100, no. 7, pp. 074510-1-074510-8, Oct. 2006.

[56] A. J. Nozik, "Multiple exciton generation in semiconductor quantum dots," Chem. Phys. Lett., vol. 457, no. 11, pp. 3-11, May 2008.

[57] M. C. Beard, J. M. Luther, O. E. Semonin, and A. J. Nozik, "Third generation photovoltaics based on multiple exciton generation in quantum confined semiconductors," Acc. Chem. Res., vol. 46, no. 6, pp. 1252-1260, June 2013.

[58] S. Singh, W. J. Jones, W. Siebrand, B. P. Stoicheff, and W. G. Schneider, "Laser generation of excitons and fluorescence in anthracene crystals," $J$. Chem. Phys., vol. 42, no. 1, pp. 330-342, Jan. 1965.

[59] D. N. Congreve et al., "External quantum efficiency above $100 \%$ in a singlet-exciton-fission-based organic photovoltaic cell," Science, vol. 340, no. 6130, pp. 334-337, Apr. 2013.

[60] B. Kraabel, D. Hulin, C. Aslangul, C. Lapersonne-Meyer, and M. Schott, "Triplet exciton generation, transport and relaxation in isolated polydiacetylene chains: Subpicosecond pump-probe experiments," Chem. Phys., vol. 227, no. 1-2, pp. 83-98, Feb. 1998.

[61] G. Lanzani et al., "Triplet exciton generation and decay in a red polydiacetylene studied by femtosecond spectroscopy," Chem. Phys. Lett., vol. 313, no. 3-4, pp. 525-532, Nov. 1999.

[62] M. Wohlgenannt, W. Graupner, R. Österbacka, G. Leising, D. Comoretto, and Z. V. Vardeny, "Singlet fission in luminescent and nonluminescent $\pi$ conjugated polymers," Synth. Met., vol. 101, no. 1-3, pp. 267-268, May 1999

[63] M. Wohlgenannt, W. Graupner, G. Leising, and Z. V. Vardeny, "Photogeneration and recombination processes of neutral and charged excitations in films of a ladder-type poly(para-phenylene)," Phys. Rev. B, vol. 60, no. 8, pp. 5321-5330, Aug. 1999.

[64] R. Österbacka, M. Wohlgenannt, M. Shkunov, D. Chinn, and Z. V. Vardeny, "Excitons, polarons, and laser action in poly( $p$-phenylene vinylene) films," J. Chem. Phys., vol. 118, no. 19, pp. 8905-8916, May 2003.

[65] A. J. Musser et al., "Activated singlet exciton fission in a semiconducting polymer," J. Am. Chem. Soc., vol. 135, no. 34, pp. 12747-12754, Aug. 2013

[66] E. Busby et al., "A design strategy for intramolecular singlet fission mediated by charge-transfer states in donor-acceptor organic materials," Nat. Mater., vol. 14, no. 4, pp. 426-433, Apr. 2015.

[67] A. Monkman, C. Rothe, S. King, and F. Dias, "Polyfluorene photophysics,"Adv. Polym. Sci., vol. 212, pp. 187-225, May 2008.

[68] F. Paquin et al., "Charge separation in semicrystalline polymeric semiconductors by photoexcitation: is the mechanism intrinsic or extrinsic?," Phys. Rev. Lett., vol. 106, no. 19, pp. 197401-1-197401-4, May 2011.

[69] X. Yang et al., "Nanoscale morphology of high-performance polymer solar cells," Nano Lett., vol. 5, no. 4, pp. 579-583, Apr. 2005.

[70] S. S. van Bavel, E. Sourty, G. de With, and J. Loos, "Three-dimensional nanoscale organization of bulk heterojunction polymer solar cells," Nano Lett., vol. 9, no. 2, pp. 507-513, Feb. 2009. 
[71] F. Etzold et al., "The effect of solvent additives on morphology and excited-state dynamics in PCPDTBT:PCBM photovoltaic blends," J. Am. Chem. Soc., vol. 134, no. 25, pp. 10569-10583, June 2012.

[72] D. E. Motaung, G. F. Malgas, and C. J. Arendse, "Correlation between the morphology and photo-physical properties of P3HT:fullerene blends," J. Mater. Sci., vol. 45, no. 12, pp. 3276-3283, June 2010.

[73] H.-Y. Chen, J. Hou, A. E. Hayden, H. Yang, K. N. Houk, and Y. Yang, "Silicon atom substitution enhances interchain packing in a thiophenebased polymer system," Adv. Mater., vol. 22, no. 3, pp. 371-375, Jan. 2010.

[74] D. Chen, A. Nakahara, D. Wei, D. Nordlund, and T. P. Russell, "P3HT/PCBM bulk heterojunction organic photovoltaics: correlating efficiency and morphology," Nano Lett., vol. 11, no. 2, pp. 561-567, Feb. 2011.

[75] T. Agostinelli et al., "The role of alkane dithiols in controlling polymer crystallization in small band gap polymer:fullerene solar cells," J. Polym. Sci., Part B: Polym. Phys., vol. 49, no. 10, pp. 717-724, May 2011.

[76] A. A. Y. Guilbert et al., "Influence of bridging atom and side chains on the structure and crystallinity of cyclopentadithiophene-benzothiadiazole polymers," Chem. Mater., vol. 26, no. 2, pp. 1226-1233, Feb. 2014.

[77] J. Nelson, "Diffusion-limited recombination in polymer-fullerene blends and its influence on photocurrent collection," Phys. Rev. B, vol. 67, no. 15, pp. 155209-1-155209-10, Apr. 2003.

[78] M. Tachiya and K. Seki, "Theory of bulk electron-hole recombination in a medium with energetic disorder," Phys. Rev. B, vol. 82, no. 8, pp. 085201-1-085201-8, Aug. 2010.

[79] C. Deibel, T. Strobel, and V. Dyakonov, "Origin of the efficient polaronpair dissociation in polymer-fullerene blends," Phys. Rev. Lett., vol. 103, no. 3, pp. 036402-1-036402-4, July 2009.

[80] X. A. Matthew, C. Beard, K. P. Knutsen, S. E. Shaheen, G. Rumbles, and R. J. Ellingson, "Photoinduced charge carrier generation in a poly(3hexylthiophene) and methanofullerene bulk heterojunction investigated by time-resolved terahertz spectroscopy," J. Phys. Chem. B, vol. 110, no. 50, pp. 25462-25471, Dec. 2006.

[81] P. D. Cunningham and L. M. Hayden, "Carrier dynamics resulting from above and below gap excitation of $\mathrm{P} 3 \mathrm{HT}$ and $\mathrm{P} 3 \mathrm{HT} / \mathrm{PCBM}$ investigated by optical-pump terahertz-probe spectroscopy," J. Phys. Chem. C, vol. 112, no. 21, pp. 7928-7935, May 2008.

[82] T. M. Burke and M. D. McGehee, "How high local charge carrier mobility and an energy cascade in a three-phase bulk heterojunction enable $>90 \%$ quantum efficiency," Adv. Mater., vol. 26, no. 12, pp. 1923-1928, Mar. 2014.

[83] F. C. Jamieson, E. B. Domingo, T. McCarthy-Ward, M. Heeney, N. Stingelin, and J. R. Durrant, "Fullerene crystallisation as a key driver of charge separation in polymer/fullerene bulk heterojunction solar cells," Chem. Sci., vol. 3, no. 2, pp. 485-492, Feb. 2011.

[84] D. H. K. Murthy, M. Gao, M. J. W. Vermeulen, L. D. A. Siebbeles, and T. J. Savenije, "Mechanism of mobile charge carrier generation in blends of conjugated polymers and fullerenes: Significance of charge delocalization and excess free energy," J. Phys. Chem. C, vol. 116, no. 16, pp. 9214-9220, Apr. 2012.

[85] S. Gélinas et al., "Ultrafast long-range charge separation in organic semiconductor photovoltaic diodes," Science, vol. 343, no. 6170, pp. 512 516, Jan. 2014

[86] B. M. Savoie et al., "Unequal partnership: Asymmetric roles of polymeric donor and fullerene acceptor in generating free charge," J. Am. Chem. Soc., vol. 136, no. 7, pp. 2876-2884, Feb. 2014.

[87] D. Credgington and J. R. Durrant, "Insights from transient optoelectronic analysis on the open-circuit voltage of organic solar cells," J. Phys. Chem. Lett., vol. 3, no. 11, pp. 1465-1478, June 2012.

[88] D. H. K. Murthy et al., "Origin of reduced bimolecular recombination in blends of conjugated polymers and fullerenes," Adv. Funct. Mater., vol. 23, no. 34, pp. 4262-4268, Sept. 2013.

[89] J. Gorenflot et al., "Nongeminate recombination in neat P3HT and P3HT:PCBM blend films," J. Appl. Phys., vol. 115, no.14, pp. 144502-1144502-9, Apr. 2014

[90] T. M. Burke, S. Sweetnam, K. Vandewal, M. D. McGehee, "Beyond Langevin recombination between free carriers and charge transfer states determines the open-circuit voltage of organic solar cells," Adv. Energy Mater., vol. 5, no. 11, pp.1500123-1-1500123-12, June 2015.

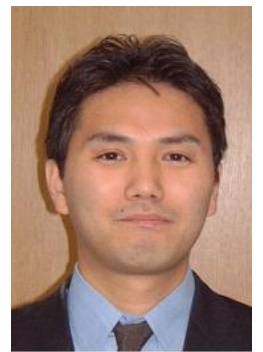

Hideo Ohkita is an Associate Professor in the Department of Polymer Chemistry at Kyoto University and concurrently a PRESTO researcher, Japan Science and Technology Agency (JST). He obtained a Doctoral degree in 1997 at Kyoto University. He became Assistant Professor in 1997 and was promoted to Associate Professor in 2006. He worked as an academic visitor with Professor Durrant at Imperial College London from 2005 to 2006. His research interests include studying photophysics and photochemistry in polymer systems. His current research focuses on spectroscopic approach to polymer solar cells

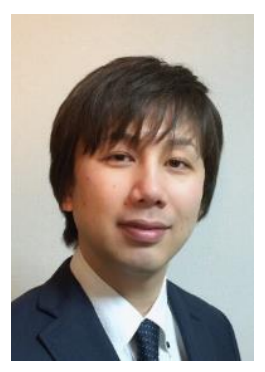

Yasunari Tamai is a Postdoctoral Fellow in the Department of Polymer Chemistry at Kyoto University. He received his Doctoral degree from Kyoto University in 2013. He worked as a Research Fellow of Japan Society for the Promotion of Science (JSPS) from 2010 to 2013. His research interests include exciton and charge dynamics in polymer systems. His current research focuses on studying photovoltaic conversion mechanisms in polymer solar cells.

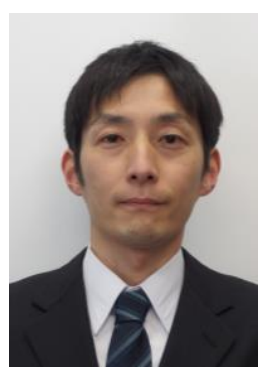

Hiroaki Benten is an Assistant Professor in the Department of Polymer Chemistry at Kyoto University. He obtained a Doctoral degree in 2005 at Kyoto University. He worked as a JSPS postdoctoral fellow at Max-Planck Institute for Polymer Research (Mainz) in the laboratory of Professor Knoll from 2006 to 2008. He became Assistant Professor in 2008. He worked as an academic visitor with Professor Ginger at University of Washington in 2013. His current research interest is in the relationship between nanoscale morphology and device performance of polymer-based solar cells.



Shinzaburo Ito is a Professor in the Department of Polymer Chemistry at Kyoto University. He obtained his doctoral degree of Engineering in 1981 from Kyoto University. He was appointed as a Research Instructor of Kyoto University in 1979. He experienced being a guest scientist of Max-Planck Institute for Polymer Research (Mainz) in the laboratory of Professor Knoll from 1991 to 1992, and was also a visiting research fellow of RIKEN (Wako) from 1993 to 1999. He was promoted to Associate Professor in 1994 and to Professor of Department of Polymer Chemistry, Kyoto University, in 1999. His research interests encompass the polymer nanostructure, polymer photophysics and photochemistry, ultrathin films and their optical and electrical properties. 